\title{
National post-colonoscopy colorectal cancer data challenge services to improve quality of colonoscopy
}

\section{다)(요 $\odot$}

\author{
Authors \\ Nicholas Burr ${ }^{1}$, Roland Valori ${ }^{2}$ \\ Institutions \\ 1 Leeds University - Cancer Epidemiology Group, Institute \\ of Cancer \& Pathology and Institute of Data Analytics, \\ Leeds, United Kingdom of Great Britain and Northern \\ Ireland \\ 2 Gloucester - Gastroenterology, Gloucester, United \\ Kingdom of Great Britain and Northern Ireland
}

\author{
Bibliography \\ DOI https://doi.org/10.1055/a-0809-5233 | \\ Endoscopy International Open 2019; 07: E728-E729 \\ (c) Georg Thieme Verlag KG Stuttgart · New York \\ eISSN 2196-9736 \\ Corresponding author \\ Roland Valori, Gloucester - Gastroenterology, Great \\ Western Road Gloucester GL1 3NN, United Kingdom of \\ Great Britain and Northern Ireland \\ Fax: +03004226892 \\ roland.valori@nhs.net
}

The presentation of colorectal cancer (CRC) months and years after a negative colonoscopy, so-called post-colonoscopy colorectal cancer (PCCRC) [1], can represent a missed opportunity for earlier diagnosis and presumably better prognosis. PCCRC has therefore become an important, perhaps the most important, quality marker for colonoscopy.

The World Endoscopy Organisation (WEO) has recently published a consensus paper on the taxonomy, investigation and reporting of PCCRC [2] aimed at both colonoscopy services and investigators. In the linked paper, Macken et al report national level data of PCCRC in Belgium, exploring characteristics of PCCRCs and factors associated with their development [3]. The Belgian investigators should be applauded for resisting the temptation to invent a new method of analyzing PCCRC rates. Instead they have followed previously published methodology for calculating rates [4] incorporating all false-negative colonoscopies and included an analysis of the method recommended by the WEO [2]. This means that we can now, for the first time, compare rates between nations and other jurisdictions as the WEO consensus guideline intended [2].

Publishing and comparing national rates of PCCRC draws attention to a problem that is otherwise "off the radar" for most endoscopists and endoscopy services. There is limited awareness of PCCRC in everyday practice because it is a relatively rare event. Furthermore, it is not easy to link cancer to previous colonoscopy because the two events are separated by months and years. It is likely that most of the time colonoscopists are unaware of a CRC that occurs several years after the index colonoscopy. There is also, perhaps, complacency that a PCCRC is a fast-growing tumor rather than a missed or incompletely re- sected lesion, or that missing lesions is something that happens to other people, or it is too rare to be concerned about. On the other hand, from the patient perspective, it is very important because it can mean the difference between having cancer or not, having treatment for later stage tumor or not, or having a worse prognosis through delayed diagnosis. Finally, there are additional costs to the health system of delayed diagnosis.

The Belgian study has shown significant variation in PCCRC rates between individuals and units. This variation supports previously published data, that to a large extent interval cancer and PCCRCs are preventable [5]. There was a greater than 10fold difference in PCCRC rates between colonoscopists. Individual physicians with low rates of colonoscopy, polyp detection and polyp resection were more likely to have PCCRCs. Unfortunately, there is limited information about characteristics of hospitals, therefore, there is no such analysis of unit-level factors that affect the rate of PCCRC, despite $30 \%$ of hospitals falling outside the $95 \%$ credible intervals on the funnel plot.

The study identified various patient- and procedure-related factors that increase the chance of PCCRC which are mostly in line with other studies, with the exception of the finding that deep sedation is associated with a lower rate of PCCRC. These consistent associations indicate that colonoscopists should be especially vigilant when performing colonoscopy for higherrisk patients and when inspecting the right colon. When views are suboptimal, or when the procedure has not achieved deep cecal intubation, there should be a definite and documented decision to repeat the test, perform another procedure or to not investigate further. This latter option may be wholly appro- 
priate taking into account patient preferences and relevant comorbidities.

Perhaps the most interesting and innovative aspect of this study was the approach to determine the prognosis for PCCRC. Here, the investigators examined survival more than 3 years after the true-positive or false-negative colonoscopy. This approach takes into account both lead-time and immortal time bias [6]. Lead-time bias can be adjusted for by measuring survival from the time point of either the false-negative or true-positive colonoscopy rather than the date of cancer diagnosis. But this does not take into account immortal time bias. This bias has the effect of improving the prognosis of PCCRC. Those undergoing a false-negative test have to survive to eventually be diagnosed with a CRC, and be labelled a PCCRC. In contrast, all deaths after a true-positive colonoscopy in which a CRC is detected will be included.

A "conditional analysis" of survival beyond 3 years, effectively eliminating these biases, demonstrated that patients with PCCRC have a significantly worse prognosis. For example, $60 \%$ of the non-PCCRC cohort lived on average 2 years longer (6.7 versus 4.7 ) than the PCCRC cohort. This would be considered an impressive difference in a trial of chemotherapy. Worse survival is what one would expect given the diagnosis is delayed, but the finding is contrary to what has been found in other studies that did not correct for these biases [7,8].

The take-home messages from this study are first that the significant variation between patients, colonoscopists and units indicates there is considerable room for improvement. This reenforces the previous literature that high-quality colonoscopy will reduce rates of interval cancer or PCCRC $[9,10]$. Second, it provides convincing evidence that if we can reduce rates of PCCRC we can reduce incidence of CRC and improve the prognosis of patients diagnosed with the disease. There is no longer any excuse for endoscopy units or individual colonoscopists to ignore the opportunity to improve survival and care of patients with colorectal cancer by improving the quality of colonoscopy.

\section{Competing interests}

Dr Valori is co-director of AnderVal Ltd, an endoscopy training company.

\section{References}

[1] Rabeneck L, Paszat LF. Circumstances in which colonoscopy misses cancer. Frontline Gastroenterol 2010; 1: $52-58$

[2] Rutter MD, Beintaris I, Valori R et al. World Endoscopy Organization Consensus Statements on Post-Colonoscopy and Post-Imaging Colorectal Cancer. Gastroenterology 2018; 155: 909-925

[3] Macken E, Van Dongen Stefan, De Brabander Isabel et al. Post-colonoscopy colorectal cancer in Belgium: characteristics and influencing factors. Endosc Int Open 2019; 07: E717-E728

[4] Morris EJA, Rutter MD, Finan PJ et al. Post-colonoscopy colorectal cancer (PCCRC) rates vary considerably depending on the method used to calculate them: a retrospective observational populationbased study of PCCRC in the English National Health Service. Gut 2014; 64: $1248-1256$

[5] Robertson D], Lieberman DA, Winawer S] et al. Colorectal cancers soon after colonoscopy: a pooled multicohort analysis. Gut 2014; 63 : 949-956

[6] Lévesque LE, Hanley JA, Kezouh A et al. Problem of immortal time bias in cohort studies: example using statins for preventing progression of diabetes. BMJ 2010; 340: doi:10.1136/bmj.b5087

[7] Samadder NJ, Curtin K, Tuohy TMF et al. Characteristics of missed or interval colorectal cancer and patient survival: a population-based study. Gastroenterology 2014; 146: 950 - 960

[8] Erichsen R, Baron JA, Stoffel EM et al. Characteristics and survival of interval and sporadic colorectal cancer patients: a nationwide population-based cohort study. Am. J. Gastroenterol 2013; 108: 1332 1340

[9] Kaminski MF, Regula J, Kraszewska E et al. Quality indicators for colonoscopy and the risk of interval cancer. N Engl J Med 2010; 362: $1795-1803$

[10] Corley DA, Jensen CD, Marks AR et al. Adenoma detection rate and risk of colorectal cancer and death. N Engl J Med 2014; 370: 1298 1306 